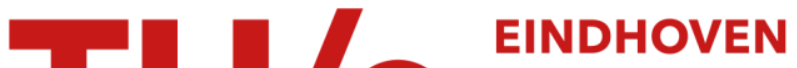

\section{Minimal LPV state-space realization driven set-membership identification}

Citation for published version (APA):

Cerone, V., Piga, D., Regruto, D., \& Toth, R. (2012). Minimal LPV state-space realization driven set-membership identification. In Proceedings of the American Control Conference (ACC 2012), $27-29$ June 2012, Montreal, Canada (pp. 3421-3426). Institute of Electrical and Electronics Engineers.

\section{Document status and date:}

Published: 01/01/2012

\section{Document Version:}

Accepted manuscript including changes made at the peer-review stage

\section{Please check the document version of this publication:}

- A submitted manuscript is the version of the article upon submission and before peer-review. There can be important differences between the submitted version and the official published version of record. People interested in the research are advised to contact the author for the final version of the publication, or visit the $\mathrm{DOI}$ to the publisher's website.

- The final author version and the galley proof are versions of the publication after peer review.

- The final published version features the final layout of the paper including the volume, issue and page numbers.

Link to publication

\section{General rights}

Copyright and moral rights for the publications made accessible in the public portal are retained by the authors and/or other copyright owners and it is a condition of accessing publications that users recognise and abide by the legal requirements associated with these rights.

- Users may download and print one copy of any publication from the public portal for the purpose of private study or research.

- You may not further distribute the material or use it for any profit-making activity or commercial gain

- You may freely distribute the URL identifying the publication in the public portal.

If the publication is distributed under the terms of Article $25 \mathrm{fa}$ of the Dutch Copyright Act, indicated by the "Taverne" license above, please follow below link for the End User Agreement:

www.tue.nl/taverne

Take down policy

If you believe that this document breaches copyright please contact us at:

openaccess@tue.nl

providing details and we will investigate your claim. 


\title{
Minimal LPV state-space realization driven set-membership identification
}

\author{
V. Cerone*, D. Piga ${ }^{\S}$, D. Regruto*, ${ }^{*}$, R. Tóth ${ }^{\S}$
}

\begin{abstract}
Set-membership identification algorithms have been recently proposed to derive linear parameter-varying (LPV) models in input-output form, under the assumption that both measurements of the output and the scheduling signals are affected by bounded noise. In order to use the identified models for controller synthesis, linear time-invariant (LTI) realization theory is usually applied to derive a statespace model whose matrices depend statically on the scheduling signals, as required by most of the LPV control synthesis techniques. Unfortunately, application of the LTI realization theory leads to an approximate state-space description of the original LPV input-output model. In order to limit the effect of the realization error, a new set-membership algorithm for identification of input/output LPV models is proposed in the paper. A suitable nonconvex optimization problem is formulated to select the model in the feasible set which minimizes a suitable measure of the state-space realization error. The solution of the identification problem is then derived by means of convex relaxation techniques.
\end{abstract}

Index Terms - Convex relaxation, LPV models, LPV realization theory, Set-membership identification.

\section{INTRODUCTION}

Linear parameter-varying (LPV) models have received a major attention from the identification and control community in the last two decades, mainly due to the strong link between gain scheduling control design and the concept of LPV control synthesis, which is now recognized as one of the most effective techniques for controlling a large class of nonlinear systems (see, e.g., the survey papers [1], [2] and the references therein). Roughly speaking, LPV models are linear systems where the coefficients of the representation (either the matrices of the state equations or the coefficients of the difference equation relating the input and the output signals) depend on some time-varying parameters/exogenous variables, commonly called scheduling signals. Real-time measurements of such signals are commonly assumed to be available. As to the identification of black-box LPV models from input-output measurements, a relevant number of approaches can be found in the literature like, for example, recursive [3] and separable [4] least squares methods, subspace identification [5], orthonormal basis function approach [6], instrumental variables methods [7] and many more. The

* V. Cerone and D. Regruto are with the Dipartimento di Automatica e Informatica, Politecnico di Torino, corso Duca degli Abruzzi 24, 10129 Torino, Italy; e-mail: vito.cerone@polito.it, diego.regruto@polito.it; Tel: +39-011-090 7064; Fax: +39-0110907198.

$\S$ D. Piga and R. Tóth are with the Delft Center for Systems and Control, Delft University of Technology, Mekelweg 2, Delft, 2628 CD, The Netherlands; email: D.Piga@tudelft.nl, R.Toth@tudelft.nl.

$\diamond$ Corresponding author readers are referred to the recent book [8] for a detailed overview of the available LPV modeling and identification approaches.

This paper focuses on a set-membership (SM) approach for the identification of LPV systems, recently proposed in [9], [10], [11], where input-output (I/O) models are considered under the assumption that both the measurements of the output and the scheduling signals are corrupted by bounded noise. In this framework, all parameters belonging to the feasible parameter set (FPS), i.e., parameters consistent with the measurements, the error bounds and the assumed model structure, are feasible solutions to the identification problem. A detailed discussion on the properties of the feasible parameter set for LPV systems given in I/O form is presented in [11], where it is shown that the FPS is a nonconvex semialgebraic set. Relaxation techniques are proposed in [9] and [11] to derive convex outer approximations of the FPS and to compute guaranteed bounds on the system parameters.

The SM identification approach has been proven to be an effective technique to obtain LPV models of complex nonlinear systems (see, e.g., the application example of a real-world automotive system in [10]). However, most LPV control synthesis approaches require models in state-space (SS) form, where the matrices describing the state-space equations depend statically on the scheduling signals. Unfortunately, exact SS realization of identified LPV I/O models requires system matrices which do not depend only on the current value of the scheduling signal but also on a number of its past samples (dynamic dependence), as proved in [12]. The problem of dynamic dependence is usually bypassed by applying the linear time-invariant (LTI) realization theory to the identified LPV I/O model (see, e.g., [13]). Although this approach has the advantage of providing a model in a form which is suitable for LPV control synthesis, the obtained SS realization is not exact, and the introduced realization error might cause significant performance degradation of the designed control system. The aim of this work is to provide a systematic way to bridge the gap between SM LPV model identification and LPV control synthesis. More precisely, a new identification algorithm is proposed to select, among the LPV I/O models belonging to the FPS, the one which minimizes a suitable measure of the state-space realization error obtained via the LTI realization theory.

The paper is organized as follows. The set-membership identification approach for LPV I/O models proposed in [9], [11] is briefly reviewed in Section II. The novel identification problem, considered in this paper, is introduced in Section III, while technical details and properties of the proposed 
identification algorithm are discussed in Section IV. A simulation example is provided in Section $\mathrm{V}$ to demonstrate the effectiveness of the presented identification scheme. The final concluding remarks are given at the end of the paper.

\section{SET-MEMBERSHIP LPV IDENTIFICATION}

Consider a SISO discrete-time LPV model described in terms of the following linear difference equations

$$
\begin{aligned}
\mathcal{A}\left(q^{-1}, \lambda(t)\right) w(t) & =\mathcal{B}\left(q^{-1}, \lambda(t)\right) u(t), \\
\mathcal{A}\left(q^{-1}, \lambda(t)\right) \eta(t) & =\mathcal{D}\left(q^{-1}, \lambda(t)\right) e(t), \\
y(t) & =w(t)+\eta(t), \\
z(t) & =\lambda(t)+\varepsilon(t),
\end{aligned}
$$

where $q^{-1}$ is the backward time-shift operator, i.e., $q^{-1} w(t)=w(t-1), u(t): \mathbb{Z} \rightarrow \mathbb{R}$ is the input signal, $w(t): \mathbb{Z} \rightarrow \mathbb{R}$ is the noise-free output signal, $y(t): \mathbb{Z} \rightarrow \mathbb{R}$ is the measured output signal, $e(t): \mathbb{Z} \rightarrow \mathbb{R}$ is a bounded noise, $\eta(t): \mathbb{Z} \rightarrow \mathbb{R}$ is the effect of the noise $e(t)$ on the measured output signal, $\lambda(t): \mathbb{Z} \rightarrow \mathbb{R}^{\mu}$ is the scheduling variable which, according to the LPV modeling and control literature (see, e.g., [1]) is assumed to be measurable, $z(t): \mathbb{Z} \rightarrow \mathbb{R}^{\mu}$ is the measured scheduling signal, while $\varepsilon(t): \mathbb{Z} \rightarrow \mathbb{R}^{\mu}$ is a bounded noise corrupting the scheduling signal measurements. In order to simplify the notation, in the sequel, the following shorthand form is adopted for a generic signal $\pi_{t} \triangleq \pi(t)$. The functions $\mathcal{A}(\cdot), \mathcal{B}(\cdot)$ and $\mathcal{D}(\cdot)$ are polynomials in the backward-shift operator $q^{-1}$ and described as

$$
\begin{aligned}
& \mathcal{A}\left(q^{-1}, \lambda_{t}\right)=1+a_{1}\left(\lambda_{t}\right) q^{-1}+\ldots+a_{n_{\mathrm{a}}}\left(\lambda_{t}\right) q^{-n_{\mathrm{a}}} \\
& \mathcal{B}\left(q^{-1}, \lambda_{t}\right)=b_{0}\left(\lambda_{t}\right)+b_{1}\left(\lambda_{t}\right) q^{-1}+\ldots+b_{n_{\mathrm{b}}}\left(\lambda_{t}\right) q^{-n_{\mathrm{b}}} \\
& \mathcal{D}\left(q^{-1}, \lambda_{t}\right)=d_{0}\left(\lambda_{t}\right)+d_{1}\left(\lambda_{t}\right) q^{-1}+\ldots+d_{n_{\mathrm{d}}}\left(\lambda_{t}\right) q^{-n_{\mathrm{d}}}
\end{aligned}
$$

where $n_{\mathrm{a}}, n_{\mathrm{b}}, n_{\mathrm{d}} \geq 0$ and the coefficients $a_{i}, b_{j}$ and $d_{l}$ are assumed to be static functions of $\lambda_{t}$, parameterized in the form

$$
\begin{gathered}
a_{i}\left(\lambda_{t}\right)=\sum_{s=1}^{n_{\phi, i}} a_{i, s} \phi_{i, s}\left(\lambda_{t}\right), \quad b_{j}\left(\lambda_{t}\right)=\sum_{s=1}^{n_{\psi, j}} b_{j, s} \psi_{j, s}\left(\lambda_{t}\right), \\
d_{l}\left(\lambda_{t}\right)=\sum_{s=1}^{n_{\sigma, l}} d_{l, s} \sigma_{l, s}\left(\lambda_{t}\right),
\end{gathered}
$$

where $\phi_{i, s}(\cdot), \psi_{j, s}(\cdot)$ and $\sigma_{l, s}(\cdot)$ are a priori chosen nonlinear basis functions bounded on $\mathbb{R}^{\mu}$. In the sequel, it is assumed that these basis functions belong to the canonical polynomial basis in the parameters $\lambda_{t}$, and we denote as $d_{\phi_{i, s}}, d_{\psi_{j, s}}$ and $d_{\sigma_{l, s}}$ the degrees of $\phi_{i, s}(\cdot), \psi_{j, s}(\cdot)$ and $\sigma_{l, s}(\cdot)$, respectively.

It is worth noting that the model considered in (1a)-(1d) is a quite general one since a number of widely adopted model structures can be obtained by properly constraining the polynomials $\mathcal{A}, \mathcal{B}$ and $\mathcal{D}$. In fact, the ARX model structure is obtained by setting $\mathcal{D}=1$, the output-error $(\mathrm{OE})$ is given by the choice $\mathcal{D}=\mathcal{A}$, while the case $\mathcal{A} \neq \mathcal{B} \neq \mathcal{D}$ leads to the more general ARMAX structure, see [8] for details.
According to the set-membership framework, the noise $e_{t}$ and each component of the noise vector $\varepsilon_{t}$ are assumed to range within given bounds $\Delta e$ and $\Delta \varepsilon_{i}$ respectively, i.e.,

$$
\begin{aligned}
e_{t} & \in \mathcal{E}_{e}=\left\{e_{t} \in \mathbb{R}:\left|e_{t}\right| \leq \Delta e\right\}, \\
\varepsilon_{t} \in \mathcal{E}_{\varepsilon} & =\left\{\varepsilon_{t} \in \mathbb{R}^{\mu}:\left|\left[\varepsilon_{t}\right]_{i}\right| \leq \Delta \varepsilon_{i}, i=1,2, \ldots, \mu\right\} .
\end{aligned}
$$

The underlaying distributions of these random variables are assumed to be arbitrary. The unknown parameter vector $\theta \in$ $\mathbb{R}^{n_{\theta}}$ to be estimated is defined as

$$
\begin{array}{r}
\theta=\left[\begin{array}{lllllll}
a_{1,1} & \ldots & a_{1, n_{\phi, 1}} \ldots & a_{n_{\mathrm{a}}, 1} & \ldots & a_{n_{\mathrm{a}}, n_{\phi, n_{\mathrm{a}}}} \\
& b_{0,1} & \ldots & b_{n_{\mathrm{b}}, n_{\psi, n_{\mathrm{b}}}} & d_{0,1} & \ldots & d_{n_{\mathrm{d}}, n_{\sigma, n_{\mathrm{d}}}}
\end{array}\right]^{\top},
\end{array}
$$

where $n_{\theta}=\sum_{i=1}^{n_{\mathrm{a}}} n_{\phi, i}+\sum_{j=0}^{n_{\mathrm{b}}} n_{\psi, j}+\sum_{l=0}^{n_{\mathrm{d}}} n_{\sigma, l}$. In the context of set-membership identification, all parameter values consistent with the collected measurements, a-priori information on the system, and the error bounds are considered as feasible solutions of the identification problem. The set of all such parameter values is called the feasible parameter set $\mathcal{D}_{\theta} \subset \mathbb{R}^{n_{\theta}}$ which is the projection on the parameter space of the set $\mathcal{D}$ of model parameters $\theta$, noise samples $e_{t}$ and $\varepsilon_{t}$ consistent with the measured data sequence in terms of the assumed model structure and error bounds. More precisely, these relations are described by equations (1a)-(1d), giving

$$
\begin{aligned}
& \mathcal{D}=\left\{(\theta, e, \varepsilon) \in \mathbb{R}^{n_{\theta}} \times \mathbb{R}^{N} \times \mathbb{R}^{\mu \times N}:\right. \\
& \mathcal{A}\left(q^{-1}, z_{t}-\varepsilon_{t}\right) y_{t}= \mathcal{B}\left(q^{-1}, z_{t}-\varepsilon_{t}\right) u_{t}+\mathcal{D}\left(q^{-1}, z_{t}-\varepsilon_{t}\right) e_{t} \\
&\left.\varepsilon_{t} \in \mathcal{E}_{\varepsilon}, e_{t} \in \mathcal{E}_{e}, t=1, \ldots, N\right\}, \quad \text { (6) }
\end{aligned}
$$

where $N$ is the length of the data sequence. Algorithms which can derive an outer bounding box of $\mathcal{D}$ are discussed in [9] and [11].

\section{STATE-SPACE REALIZATION-ORIENTED SELECTION} OF A SINGLE MODEL FROM THE FEASIBLE SET

The mainstream LPV control synthesis techniques available in literature are based on the following state-space representation:

$$
\begin{aligned}
x(t+1) & =A\left(\lambda_{t}\right) x(t)+B\left(\lambda_{t}\right) u(t), \\
w(t) & =C\left(\lambda_{t}\right) x(t)+D\left(\lambda_{t}\right) u(t),
\end{aligned}
$$

where the entries of matrices $A, B, C, D$ are static functions of the scheduling parameter $\lambda$. As the feasible parameter set $\mathcal{D}_{\theta}$ is defined w.r.t. the LPV input-output (IO) representation (1a), the identified LPV-IO model can be used for control synthesis, provided that it is converted into an equivalent SS representation. Unfortunately, as shown in [12], exact state-space realization leads to an LPV-SS model with a dynamic dependence of $A, B, C, D$ on $\lambda$, thus increasing complexity of the control synthesis. Indeed, an approximate SS realization of the I/O model (1a) with static dependence of $A, B, C, D$ on the scheduling parameter is to be preferred in order to use common LPV control design tools [14].

The LPV-IO model (1a) with $n_{\mathrm{a}} \geq n_{\mathrm{b}}$ is usually converted to the so-called companion-reachability canonical SS representation given by (7), where $d=b_{0}$ and $c_{j}=b_{j}-a_{j} b_{0}$ 


$$
\left[\begin{array}{l|l}
A\left(\lambda_{t}\right) & B\left(\lambda_{t}\right) \\
\hline C\left(\lambda_{t}\right) & D\left(\lambda_{t}\right)
\end{array}\right]=\left[\begin{array}{ccccc|c}
-a_{1}\left(\lambda_{t}\right) & -a_{1}\left(\lambda_{t}\right) & \ldots & -a_{n_{\mathrm{a}}-1}\left(\lambda_{t}\right) & -a_{n_{\mathrm{a}}}\left(\lambda_{t}\right) & 1 \\
1 & 0 & \ldots & \ldots & 0 & 0 \\
0 & \ddots & \ddots & \ddots & 0 & \vdots \\
\vdots & \ddots & \ddots & 0 & \vdots & \vdots \\
0 & \ldots & 0 & 1 & 0 & 0 \\
\hline c_{1}\left(\lambda_{t}\right) & \cdots & \cdots & \cdots & c_{n_{\mathrm{a}}}\left(\lambda_{t}\right) & d\left(\lambda_{t}\right)
\end{array}\right]
$$

and when $n_{\mathrm{b}}<n_{\mathrm{a}}$, then $b_{j}=0$ for $j>n_{\mathrm{b}}$. Indeed, because of the static dependence of matrixes $A, B, C, D$ on the scheduling parameter, this is only an approximation of the true I/O realization (1a) (see [8]). Note that using a nonminimal SS "realization" with a state vector

$x(t)=\left[\begin{array}{llllll}y(t-1) & \ldots & y\left(t-n_{\mathrm{a}}\right) & u(t-1) & \ldots & u\left(t-n_{\mathrm{b}}\right)\end{array}\right]^{\top}$

avoids dynamic dependency, but on the expense of alterating the behavior of the system by introducing a delay.

In [14], the following index $J$ is proposed to measure the approximation error of LTI-type realizations like (7):

$$
J=\sup _{\lambda \in \mathcal{P}}\left\|\left[\begin{array}{llll}
g_{0} & \ldots & g_{n_{\mathrm{a}}}
\end{array}\right]^{\top}-\left[\begin{array}{lll}
\hat{g}_{0} & \ldots & \hat{g}_{\mathrm{n}_{\mathrm{a}}}
\end{array}\right]^{\top}\right\|_{\infty},
$$

where $\left\{g_{i}\right\}_{i=0}^{n_{\mathrm{a}}}$ are the first $n_{\mathrm{a}}$ Markov parameters of the exact state-space realization, while $\left\{\hat{g}_{i}\right\}_{i=0}^{n_{\mathrm{a}}}$ are the Markov parameters corresponding to the approximate LTI-like SSrealization and $\mathcal{P} \in \mathbb{P}^{\mathbb{Z}}$ is the set of admissible trajectories of $\lambda$. Therefore, in order to derive a suitable model for controller design, we select the parameter value $\theta^{*} \in \mathcal{D}_{\theta}$ which minimizes the worst-case approximation error in terms of $J$.

\section{COMputation of $\theta^{*}$ AND RELATED Discussion}

Let us first define the set $\mathcal{P}_{\text {id }}$ of all the trajectories (and their time-shifted copies) taken by the scheduling parameter $\lambda$ during the experiments performed to collect data for identification. Then, $\theta^{*}$ can be computed by solving the following optimization problem:

$$
\theta_{\text {id }}^{*}=\arg \min _{\theta \in \mathcal{D}_{\theta}} J_{\text {id }}^{p},
$$

where $J_{\text {id }}^{p}$ can be either

$$
J_{\text {id }}^{\infty}=\sup _{\lambda \in \mathcal{P}_{\text {id }}}\left\|\left[\begin{array}{lll}
g_{0} & \ldots & g_{n_{\mathrm{a}}}
\end{array}\right]^{\top}-\left[\begin{array}{lll}
\hat{g}_{0} & \ldots & \hat{g}_{n_{\mathrm{a}}}
\end{array}\right]^{\top}\right\|_{\infty},
$$

for $p=\infty$ or

$$
J_{\mathrm{id}}^{p}=\sup _{\lambda \in \mathcal{P}_{\mathrm{id}}}\left\|\left[\begin{array}{lll}
g_{0} & \ldots & g_{n_{\mathrm{a}}}
\end{array}\right]^{\top}-\left[\begin{array}{lll}
\hat{g}_{0} & \ldots & \hat{g}_{n_{\mathrm{a}}}
\end{array}\right]^{\top}\right\|_{p}^{p},
$$

where $\|\cdot\|_{p}$ can be any $p$-norm with $p=1,2, \ldots$. Let us denote the minimum of the functional $J_{\text {id }}^{p}\left(\theta_{\text {id }}^{*}\right)$ as $\widetilde{J}_{\text {id }}^{p}$. Problem (10) is a polynomial optimization problem since it enjoys the following properties:

Property 1: The functionals $J_{\mathrm{id}}^{\infty}$ and $J_{\mathrm{id}}^{p}$ are polynomial functions of $\theta$.

Property 2: The constraints defining the FPS $\mathcal{D}_{\theta}$ are polynomial functions in the unknown model parameters $\theta$ and noise samples $e_{t}$ and $\varepsilon_{t}$.
Property 1 follows from the definition of the Markov parameters $g_{0}, \ldots, g_{n_{\mathrm{a}}}$ (see [14] for details), while Property 2 follows from the structure of the set $\mathcal{D}$ in (6) (see [9], [11] for details).

Although problem (10) is nonconvex in general, a hierarchy of relaxed semidefinite (SDP) programming problems can be constructed (in the spirit of [15]) whose optima are guaranteed to converge to the global optimum of (10). Solution of such relaxed problems can be computed by using standard SDP solvers like SeDuMi [16].

Remark 1: Although the proposed convex relaxation approach is guaranteed to converge to the global minimum of the original nonconvex problem (10), its implementation is computationally intractable for problems with medium to large dimension. However, accepting a certain degree of conservativeness in the computation of the minimum $\widetilde{J}_{\mathrm{id}}^{p}$, the computational burden can be significantly reduced by using the convex outer approximations of $\mathcal{D}$ proposed in [9] and [11].

Remark 2: The particular model associated with the identified parameter $\theta_{\text {id }}^{*}$ guarantees the minimum worst case realization error $J_{\text {id }}$ computed over the set of the scheduling parameter trajectories $\mathcal{P}_{\text {id }}$ covered in the collected data. Given $\theta_{\mathrm{id}}^{*}$ we can formulate the problem of evaluating the worst-case realization error $J$ corresponding to such a parameter over the set of all scheduling parameter trajectories of finite length $\left[\lambda(t) \lambda(t-1) \ldots \lambda\left(t-n_{\mathrm{a}}\right)\right] \in \Lambda \subset \mathbb{R}^{n_{\mathrm{a}}+1}$, where $\Lambda$ is an hypercube in the space of the finite scheduling sequences $\left[\begin{array}{lllll}\lambda(t) & \lambda(t-1) & \ldots & \lambda\left(t-n_{\mathrm{a}}\right)\end{array}\right]$. The problem can be written as follows:

$$
J_{\mathrm{wc}}^{p}=\sup _{\lambda \in \Lambda}\left\|\left[\begin{array}{lll}
g_{0} & \ldots & g_{n_{\mathrm{a}}}
\end{array}\right]^{\top}-\left[\begin{array}{lll}
\hat{g}_{0} & \ldots & \hat{g}_{n_{\mathrm{a}}}
\end{array}\right]^{\top}\right\|_{p}^{p},
$$

where the Markov parameters corresponding to both the exact and the approximated LTI-like state-space realization are computed for $\theta=\theta_{\mathrm{id}}^{*}$. Because of the polynomial objective function, (13) is again a semialgebraic optimization problem whose global optimum $J_{\text {wc }}$ can be approximated through the SDP relaxations discussed above. In this way, an upper bound on the global optimum $J_{\mathrm{wc}}$ can be computed. Such an upper bound complements the lower bound computed by means of the gridding approach proposed in [14].

On the basis of the properties of $\mathcal{D}$, we can also compute an upper bound on the simulation error of the identified SSLPV model. Denote by $y=\left[\begin{array}{llll}y_{1} & y_{2} & \ldots & y_{N}\end{array}\right]$ the output sequence collected in the identification experiment and by $w=\left[\begin{array}{llll}w_{1} & w_{2} & \ldots & w_{N}\end{array}\right]$ the corresponding noise-free sequence. Define $\hat{w}_{\mathrm{SS}}$ as the output sequence of the LTI-like statespace realization of the identified I/O system (corresponding 
to $\theta_{\mathrm{id}}^{*}$ ). Assume that $\hat{w}_{\mathrm{SS}}$ is obtained by simulating the state-space system under the same conditions considered in the identification experiment (i.e. same input sequence $u=$ $\left[\begin{array}{llll}u_{1} & u_{2} & \ldots & u_{N}\end{array}\right]$, zero initial conditions and same scheduling parameter trajectories). The following results holds.

Result 1: Assume that the system to be identified is asymptotically stable and belongs to the model class described by equations (1a)-(1d) with $\mathcal{D}=1$ (OE structure). The following statements hold:

R1: $\quad\left\|w-\hat{w}_{\mathrm{SS}}\right\|_{\infty} \leq 2 \Delta e+\widetilde{J}_{\mathrm{id}}^{\infty}\|u\|_{1}+\gamma_{\infty}\|u\|_{1}$

R2: $\quad\left\|w-\hat{w}_{\text {SS }}\right\|_{\infty} \leq 2 \Delta e+\widetilde{J}_{\text {id }}^{1}\|u\|_{\infty}+\gamma_{1}\|u\|_{\infty}$

where $\gamma_{\infty} \geq 0$ and $\gamma_{1} \geq 0$ are bounded coefficients determined by the LPV system associated with $\theta_{\text {id }}^{*}$ and the approximation error $\widetilde{J}_{\text {id }}^{\infty}$ and $\widetilde{J}_{\text {id }}^{1}$ respectively.

\section{Proof}

$$
\begin{aligned}
\left\|w-\hat{w}_{\mathrm{SS}}\right\|_{\infty} & =\left\|y-e-\hat{w}_{\mathrm{SS}}\right\|_{\infty} \\
& \leq\left\|y-\hat{w}_{\mathrm{SS}}\right\|_{\infty}+\Delta e \\
& =\left\|y-\hat{w}_{\mathrm{IO}}+\hat{w}_{\mathrm{IO}}-\hat{w}_{\mathrm{SS}}\right\|_{\infty}+\Delta e \\
& \leq\left\|y-\hat{w}_{\mathrm{IO}}\right\|_{\infty}+\left\|\hat{w}_{\mathrm{IO}}-\hat{w}_{\mathrm{SS}}\right\|_{\infty}+\Delta e \\
& \leq 2 \Delta e+\left\|\hat{w}_{\mathrm{IO}}-\hat{w}_{\mathrm{SS}}\right\|_{\infty} .
\end{aligned}
$$

Now let us assume, without loss of generality, that $\| \hat{w}_{\mathrm{IO}}-$ $\hat{w}_{\mathrm{SS}} \|_{\infty}=\left|\hat{w}_{\mathrm{IO}}(t)-\hat{w}_{\mathrm{SS}}(t)\right|$, i.e., the $\mathrm{t}$-th element of the vector $\hat{w}_{\mathrm{IO}}-\hat{w}_{\mathrm{SS}}=\left[\hat{w}_{\mathrm{IO}}(1)-\hat{w}_{\mathrm{SS}}(1) \hat{w}_{\mathrm{IO}}(2)-\right.$ $\left.\hat{w}_{\mathrm{SS}}(2) \ldots \hat{w}_{\mathrm{IO}}(N)-\hat{w}_{\mathrm{SS}}(N)\right]$ is the one which has the maximum absolute value. Indeed,

$$
\left|\hat{w}_{\mathrm{IO}}(t)-\hat{w}_{\mathrm{SS}}(t)\right|=\left|\sum_{l=0}^{t}\left(g_{l}(t)-\hat{g}_{l}(t)\right) u(t-l)\right| .
$$

The latter can also be written as $\left|\Delta_{0}^{t} g(t)[u(t) \ldots u(0)]^{\top}\right|$ by introducing the following operator:

$$
\Delta_{\tau}^{s} g(t) \doteq\left[g_{\tau}(t)-\hat{g}_{\tau}(t) \ldots g_{s}(t)-\hat{g}_{s}(t)\right]
$$

with $s \geq \tau \geq 0$. By using Hölders' inequality (see, e.g., [17]):

$$
\left|\Delta_{0}^{t} g(t)[u(t) \ldots u(0)]^{\top}\right| \leq\left\|\Delta_{0}^{t} g(t)\right\|_{\infty}\|[u(t) \ldots u(0)]\|_{1}
$$

Since by construction $\left\|\Delta_{0}^{t} g(t)\right\|_{\infty} \leq \widetilde{J}_{\text {id }}^{\infty}$ if $t \leq n_{\mathrm{a}}$, we get

$$
\left|\Delta_{0}^{t} g(t)[u(t) \ldots u(0)]^{\top}\right| \leq \widetilde{J}_{\mathrm{id}}^{\infty}\|u\|_{1} .
$$

In case $t>n_{\mathrm{a}}$, then

$$
\left\|\Delta_{0}^{t} g(t)\right\|_{\infty} \leq\left\|\Delta_{0}^{n_{\mathrm{a}}} g(t)\right\|_{\infty}+\left\|\Delta_{n_{\mathrm{a}}+1}^{N} g(t)\right\|_{\infty},
$$

as there are at most $N>0$ data points. Following the same reasoning, it can be concluded that

$$
\left|\Delta_{0}^{t} g(t)[u(0) \ldots u(t)]^{\top}\right| \leq \widetilde{J}_{\mathrm{id}}^{\infty}\|u\|_{1}+\left\|\Delta_{n_{\mathrm{a}}+1}^{N} g(t)\right\|_{\infty}\|u\|_{1} .
$$

Denote $\left\|\Delta_{n_{\mathrm{a}}+1}^{N} g(t)\right\|_{\infty}$ as $\gamma_{\infty}$. As the impulse responses $\left\{g_{i}(t)\right\}_{i=0}^{\infty}$ and $\left\{\hat{g}_{i}(t)\right\}_{i=0}^{\infty}$ belong to an asymptotically stable LPV system, hence there exist finite real numbers $\alpha, \hat{\alpha}, \beta, \hat{\beta}>0$, such that for all possible scheduling trajectories $\lambda \in\left(\mathbb{R}^{\mu}\right)^{\mathbb{Z}}, i \in \mathbb{Z}_{0}^{+}$and $t \in \mathbb{Z}$ :

$$
\left|g_{i}(t)\right| \leq \beta e^{-\frac{1}{\alpha} i}, \quad\left|\hat{g}_{i}(t)\right| \leq \hat{\beta} e^{-\frac{1}{\hat{\alpha}} i} .
$$

Consider that $\alpha, \hat{\alpha}, \beta$ and $\hat{\beta}$ are the minimum values such that the above inequalities are satisfied. In this case $\alpha$ and $\hat{\alpha}$ correspond to the worst-case "time constants" of the corresponding systems and are determined by the first $n_{\mathrm{a}}+1$ Markov parameters, i.e., $\left\{g_{i}(t)\right\}_{i=0}^{n_{\mathrm{a}}}$ and $\left\{\hat{g}_{i}(t)\right\}_{i=0}^{n_{\mathrm{a}}}$. The latter means that in case of a good approximation of $\left\{g_{i}(t)\right\}_{i=0}^{n_{\mathrm{a}}}$ by $\left\{\hat{g}_{i}(t)\right\}_{i=0}^{n_{\mathrm{a}}}$, these bounding parameters must be very close, i.e., $\beta e^{-\frac{1}{\alpha} i} \approx \beta e^{-\frac{1}{\alpha} i}$. From (21) it follows that

$$
0 \leq\left|\Delta_{i}^{i} g(t)\right| \leq \beta e^{-i / \alpha}+\hat{\beta} e^{-i / \hat{\alpha}} \approx 2 \beta e^{-i / \alpha}
$$

which gives

$$
\left\|\Delta_{n_{\mathrm{a}}+1}^{N} g(t)\right\|_{\infty}=\gamma_{\infty} \leq \beta e^{-n_{\mathrm{a}} / \alpha}+\hat{\beta} e^{-n_{\mathrm{a}} / \hat{\alpha}} \approx 2 \beta e^{-n_{\mathrm{a}} / \alpha} .
$$

Equation (23) proves that $\gamma_{\infty}$ is bounded. Therefore, from (20) and (23), result $\mathbf{R} \mathbf{1}$ follows.

The $\mathbf{R} 2$ case, is just a variation of the Hölders' inequality, where

$$
\underbrace{\left\|\Delta_{n_{\mathrm{a}}+1}^{N} g(t)\right\|_{1}}_{\gamma_{1}} \leq \sum_{i=n_{\mathrm{a}}+1}^{N} 2 \beta e^{-\frac{i}{\alpha}}=2 \beta \frac{\left(e^{-\frac{n_{\mathrm{a}}+1}{\alpha}}-e^{-\frac{N}{\alpha}}\right)}{e^{\frac{1}{\alpha}}-1}
$$

Furthermore, we know that for $i=0, \ldots, n_{\mathrm{a}}$ :

$$
\widetilde{J}_{\text {id }}^{1} \leq 2 \beta \frac{1-e^{-\frac{i+1}{\alpha}}}{1-e^{-\frac{1}{\alpha}}}
$$

giving

$$
\widetilde{J}_{\mathrm{id}}^{1} \leq 2 \beta \frac{1-e^{-\frac{n_{\mathrm{a}}+1}{\alpha}}}{1-e^{-\frac{1}{\alpha}}} .
$$

From which it follows that

$$
1-\widetilde{J}_{\mathrm{id}}^{1} \frac{1-e^{-\frac{1}{\alpha}}}{2 \beta} \geq e^{-\frac{n_{\mathrm{a}}+1}{\alpha}},
$$

which via (24) implies that $\gamma_{1}$ is bounded by

$$
\gamma_{1} \leq 2 \beta+\widetilde{J}_{\mathrm{id}}^{1} \frac{1-e^{-\frac{1}{\alpha}}}{1-e^{\frac{1}{\alpha}}} .
$$

Remark 3: This result states that the simulation error between the true output of the system to be identified and the output of the approximate LTI-like state-space realization of the identified model is bounded by a given constant which depends on the input sequence, the noise bound and the minimum of the functional $J_{\mathrm{id}}^{p}$.

Remark 4: Given the input sequence $u$ one can decide if it is better to minimize, in the identification stage, $J_{\text {id }}^{\infty}$ (case $\|u\|_{1}<\|u\|_{\infty}$ ) or $J_{\text {id }}^{1}$ (case $\|u\|_{\infty}<\|u\|_{1}$ ). 


\section{Simulation Example}

In this section we demonstrate the performance of the proposed identification scheme on a relevant simulation example taken from [18].

Consider the mechanical system depicted in Fig. 1 consisting of a mass connected to a spring and a varying damper. This problem is one of the typical phenomena occurring in the motion control of many mechatronic systems like in active suspension. Denote $x$ the position (in $[\mathrm{m}]$ ) of the mass $m$ (in $[\mathrm{kg}]), \mathrm{k}_{\mathrm{s}}>0$ (in $\left.[\mathrm{N} / \mathrm{m}]\right)$ the stiffness of the spring and $c_{\mathrm{d}}>0$ (in $[\mathrm{Ns} / \mathrm{m}]$ ) the varying damping. Introduce $F$ as the force (in $[\mathrm{N}]$ ) acting on the mass $m$. By using the Newton-Euler force balance approach and by considering $F$ as the input $u, w$ as the displacement with respect to the position corresponding to input $F=0$ and $\lambda \in[0,1]$ as the scheduling variable such that $c_{d}=c_{d}^{(0)}+c_{d}^{(1)} \lambda$, the mechanical system in Fig. 1 can be written as a continuous time LPV model. Then, by using a simple backward Euler type of discretization in a zero-order-hold setting with sampling period $T_{\mathrm{d}}>0$, the following discrete-time (DT) LPV representation for the considered system is obtained:

$$
\begin{array}{r}
w(t)=\frac{T_{\mathrm{d}} \mathrm{c}_{\mathrm{d}}^{(1)} \lambda_{t}+T_{\mathrm{d}} \mathrm{c}_{\mathrm{d}}^{(0)}+2 m}{L\left(\lambda_{t}\right)} w(t-1)-\frac{m}{L\left(\lambda_{t}\right)} w(t-2)+ \\
+\frac{T_{\mathrm{d}}^{2}}{L\left(\lambda_{t}\right)} u(t),
\end{array}
$$

where $L\left(\lambda_{t}\right)=T_{\mathrm{d}} \mathrm{c}_{\mathrm{d}}^{(1)} \lambda_{t}+T_{\mathrm{d}} \mathrm{c}_{\mathrm{d}}^{(0)}+m+\mathrm{t}_{\mathrm{s}} T_{\mathrm{d}}^{2}$. The reader is referred to [18] for further details on the derivation of equation (29). In order to simplify the problem, we approximate $\frac{1}{L}$ by its $1^{\text {st }}$-order Taylor expansion at the mid-point of $\mathbb{P}$, i.e. at $\lambda_{t}=0.5$ :

$$
\left.\frac{1}{L\left(\lambda_{t}\right)}\right|_{\lambda_{t}=0.5} \approx \frac{1}{\frac{\tau_{1}}{2}+\tau_{0}}-\frac{\tau_{1}}{\left(\frac{\tau_{1}}{2}+\tau_{0}\right)^{2}}\left(\lambda_{t}-0.5\right),
$$

where $\tau_{0}=T_{\mathrm{d}} \mathrm{c}_{\mathrm{d}}^{(0)}+m+\mathrm{k}_{\mathrm{s}} T_{\mathrm{d}}^{2}$ and $\tau_{1}=T_{\mathrm{d}} \mathrm{c}_{\mathrm{d}}^{(1)}$. Then the resulting DT-LPV representation reads as

$$
\begin{aligned}
w(t)=- & \left(a_{10}+a_{11} \lambda_{t}+a_{12} \lambda_{t}^{2}\right) w(t-1)- \\
& \left(a_{20}+a_{21} \lambda_{t}\right) w(t-2)+\left(b_{00}+b_{01} \lambda_{t}\right) u(t),
\end{aligned}
$$

where $a_{11}=-\tau^{\prime} T_{\mathrm{d}} \mathrm{c}_{\mathrm{d}}^{(1)}+\tau^{\prime \prime}\left(T_{\mathrm{d}} \mathrm{c}_{\mathrm{d}}^{(0)}+2 m\right), a_{10}=$ $-\tau^{\prime}\left(T_{\mathrm{d}} \mathrm{c}_{\mathrm{d}}^{(0)}+2 m\right), a_{12}=\tau^{\prime \prime} T_{\mathrm{d}} \mathrm{c}_{\mathrm{d}}^{(1)}, a_{20}=\tau^{\prime} m, a_{21}=$ $-\tau^{\prime \prime} m, b_{00}=\tau^{\prime} T_{\mathrm{d}}^{2}, b_{01}=-\tau^{\prime \prime} T_{\mathrm{d}}^{2}$ with $\tau^{\prime}=\frac{2}{\tau_{1}+2 \tau_{0}}+$ $\frac{2 \tau_{1}}{\left(\tau_{1}+2 \tau_{0}\right)^{2}}$ and $\tau^{\prime \prime}=\frac{4 \tau_{1}}{\left(\tau_{1}+2 \tau_{0}\right)^{2}}$. The numerical values of the system parameters used in the example are: $T_{d}=0.05$ $\mathrm{s}, m=0.01 \mathrm{~kg}, k_{s}=0.85 \mathrm{~N} / \mathrm{m}, c_{d}^{(1)}=c_{d}^{(0)}=0.5$ $\mathrm{Ns} / \mathrm{m}$. In the sequel, both the input signal $u(t)$ and the scheduling parameter $\lambda_{t}$ are considered as random variables uniformly distributed between $[-0.5 ; 0.5]$ and $[0.05 ; 0.95]$ respectively. Realization of these signals are used to generate a sequence of output data with length $N=50$ that will be exploited to identify the unknown LPV-model parameters $\theta=\left[\begin{array}{lllllll}a_{10} & a_{11} & a_{12} & a_{20} & a_{21} & b_{00} & b_{01}\end{array}\right]^{\top}$. The noisefree output signal $w(t)$ and the scheduling parameter $\lambda_{t}$ are corrupted by measurement noises $e(t)$ and $\varepsilon(t)$ bounded in the intervals $[-\Delta e ; \Delta e]$ and $[-\Delta \varepsilon ; \Delta \varepsilon]$, respectively. An output error structure is assumed for the noise model. The

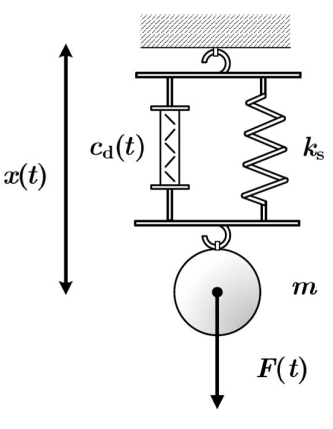

Fig. 1. Mass-spring-damper system with varying damping.

chosen error bounds $\Delta e$ and $\Delta \varepsilon$ are such that the signal to noise ratios on the output $\mathrm{SNR}_{w}$ and on the scheduling signal $\mathrm{SNR}_{\lambda}$, defined as:

$$
\begin{aligned}
& \mathrm{SNR}_{w}=10 \log _{10} \frac{\|w(t)\|_{2}^{2}}{\|e(t)\|_{2}^{2}}, \\
& \mathrm{SNR}_{\lambda}=10 \log _{10} \frac{\|\lambda(t)\|_{2}^{2}}{\|\varepsilon(t)\|_{2}^{2}},
\end{aligned}
$$

are equal to $27 \mathrm{~dB}$ and $29 \mathrm{~dB}$, respectively. First, LPV parameters $\theta_{\text {id }}^{*}$ are identified through the discussed method. In particular, solution to the nonconvex optimization problem (10) for $p=\infty$ is computed by relaxing (10) by means of the LMI-relaxation techniques proposed in [15]. The open source software Gloptipoly by [19], has been used to convert the original identification problem (10) into a hierarchy of convex SDP problems. Then, in order to show the effectiveness of the presented method, an outer bounding box of the FPS $\mathcal{D}_{\theta}$ is derived by using the algorithm proposed in [9] and, among all the parameters $\theta$ belonging to such a box, the central value $\theta^{c}$ is chosen as the output of the identification process (see, e.g., [20] for a detailed discussion of the optimality properties enjoyed by the central estimate in the set-membership context).

A collection of 50 input/output data, that are not exploited during the identification, is used to evaluate the accuracy of the LTI-like SS-realizations of the identified LPV I/O models. The comparison between the true output data $w(t)$ and the estimate output signal $\hat{w}(t)$ obtained by simulating the LTI-like SS-realizations of the LPV I/O models with identified parameters $\theta_{\text {id }}^{*}$ and $\theta^{c}$ is reported in Fig. 2. The following error measures:

$$
\begin{gathered}
\mathrm{SE}_{\infty}=\frac{\|(w(t)-\hat{w}(t))\|_{\infty}}{\|(w(t))\|_{\infty}} \\
\mathrm{SE}_{p}=\frac{\|(w(t)-\hat{w}(t))\|_{p}^{p}}{\|(w(t))\|_{p}^{p}}, \text { for } p=1,2
\end{gathered}
$$

are computed to quantify the error between the true output $w(t)$ and the estimated output $\hat{w}(t)$. The obtained values are reported in Table I. Results reported in Fig. 2 and Table I show that the SS-realization of the I/O model with parameter $\theta_{\text {id }}^{*}$ is significantly more accurate than the SS-realization of the model with central parameter $\theta^{c}$. 


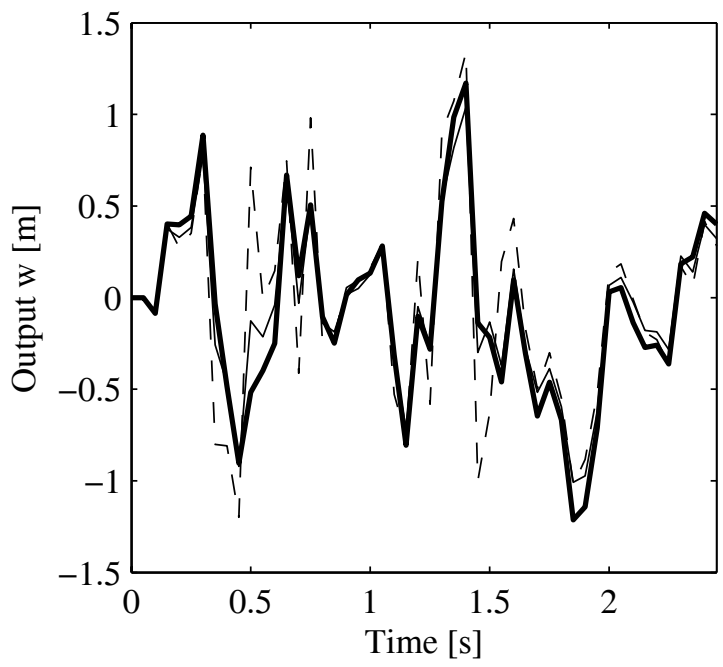

Fig. 2. Comparison between LTI-like SS-realizations of I/O-LPV models identified with parameters $\theta_{\mathrm{id}}^{*}$ and $\theta^{c}$. True output signal (solid thick line), estimated data from parameters $\theta_{\mathrm{id}}^{*}$, (solid thin line), estimated data from central estimate $\theta^{c}$ (dashed line).

\section{TABLE I}

Measures $\mathrm{SE}_{2}, \mathrm{SE}_{1}$ AND $\mathrm{SE}_{\infty}$ OF THE ERROR BETWEen true OUTPUT SIGNAL $w(t)$ AND ESTIMATED OUTPUT SIGNAL $\hat{w}(t)$ OBTAINED BY SIMULATING THE LTI-LIKE SS-REALIZATIONS OF THE I/O-LPV MODELS WITH IDENTIFIED PARAMETERS $\theta_{\text {id }}^{*}$ AND $\theta^{c}$.

\begin{tabular}{cccc}
\hline $\begin{array}{c}\text { Identified } \\
\text { parameter }\end{array}$ & $\mathrm{SE}_{2}$ & $\mathrm{SE}_{1}$ & $\mathrm{SE}_{\infty}$ \\
\hline$\theta_{\mathrm{id}}^{*}$ & 0.22 & 0.04 & 0.64 \\
\hline$\theta^{c}$ & 0.65 & 0.55 & 1.01 \\
\hline
\end{tabular}

\section{CONCLUSION}

A novel set-membership approach for the identification of LPV I/O models is presented in the paper. The problem of computing the model in the feasible parameter set which minimizes the approximation error of applying LTI statespace realization theory on it is formulated in terms of semialgebraic optimization and solved by means of suitable convex relaxation techniques. The simulation error between the true output of the data-generating system and the simulated output of the state-space realization of the identified model is shown to be bounded by a constant which depends on the input sequence, the identified model and a suitable measure of the approximation error introduced by the realization stage. The reported simulated example shows the effectiveness of the proposed approach.

\section{ACKNOWLEDGMENTS}

This research was mainly developed when Dr. D. Piga was a Ph.D student at the Politecnico di Torino and concluded when he was a postdoc at the Delft University of Technology.

\section{REFERENCES}

[1] W. J. Rugh and J. S. Shamma, "Research on gain scheduling," Automatica, vol. 36, no. 10, pp. 1401-1425, 2000
[2] D. J. Leith and W. E. Leithead, "Survey of gain-scheduling analysis and design," International Journal of Control, vol. 73, pp. 1001-1025, 2000

[3] B. A. Bamieh and L. Giarré, "Identification of linear parameter-varying models," International Journal of Robust Nonlinear Control, vol. 12, no. 9, pp. 841-853, 2002.

[4] F. Previdi and M. Lovera, "Identification of non-linear parametrically varying models using separable least squares," International Journal of Control, vol. 77, no. 16, pp. 1382-1392, 2004.

[5] F. Felici, J. W. V. Wingerden, and M. Verhaegen, "Subspace identification of MIMO LPV systems using a periodic scheduling sequence,' Automatica, vol. 43, pp. 1684-1697, 2007.

[6] R. Tóth, P. S. C. Heuberger, and P. M. J. Van den Hof, "Asymptotically optimal orthonormal basis functions for LPV system identification," Automatica, vol. 45, no. 6, pp. 1359-1370, 2010.

[7] V. Laurain, M. Gilson, R. Tóth, and H. Garnier, "Refined instrumental variable methods for identification of LPV Box-Jenkins models," Automatica, vol. 46, no. 6, pp. 959-967, 2010.

[8] R. Tóth, Modeling and identification of linear parameter-varying systems. Springer, 2010.

[9] V. Cerone and D. Regruto, "Set-membership identification of LPV models with uncertain measurements of the time-varying parameter," in Proc. of the 47th IEEE Conference on Decision and Control, 2008, pp. 4491-4496.

[10] V. Cerone, D. Piga, and D. Regruto, "Set-membership LPV model identification of vehicle lateral dynamics," Automatica, vol. 47, no. 8 , pp. 1794-1799, 2011.

[11] _ "Convex relaxation techniques for set-membership identification of LPV systems," in Proc. of the American Control Conference 2011, San Francisco, California (USA), 2011.

[12] R. Tóth, J. C. Willems, P. S. C. Heuberger, and P. M. J. Van den Hof "The behavioral approach to linear parameter-varying systems," IEEE Trans. Automatic Control, vol. 56, no. 11, pp. 2499-2514, 2011.

[13] M. G. Wassik, M. van de Wal, C. W. Scherer, and O. Bosgra, "LPV control for a wafer stage: Beyond the theoretical solution," Control Engineering Practice, vol. 13, no. 2, pp. 231-245, 2004.

[14] R. Tóth, H. S. Abbas, and H. Werner, "On the state-space realization of LPV input-output models: practical approaches," IEEE Trans. Control Systems Technology, vol. 20, no. 1, pp. 139-153, 2012.

[15] J. B. Lasserre, "Global optimization with polynomials and the problem of moments," SIAM Journal on Optimization, vol. 11, pp. 796-817, 2001.

[16] J. F. Sturm, "Using SeDuMi 1.02, a MATLAB Toolbox for optimization over symmetric cones," Optim. Methods Software, vol. 11, no. 12, pp. 625-653, 1999.

[17] G. Golub and C. van Loan, Matrix computations, Third edition. London: The Johns Hopkins University Press, 1996.

[18] R. Tóth, V. Laurain, M. Gilson, and H. Garnier, "Instrumental variable scheme for closed-loop LPV model identification," In print, Automatica, 2012.

[19] D. Henrion and J. B. Lasserre, "Gloptipoly: Global optimization over polynomials with Matlab and SeDuMi," ACM Transactions Math. Soft, vol. 29, pp. 165-194, 2003.

[20] B. Z. Kacewicz, M. Milanese, and A. Vicino, "Optimality of central and projection algorithms," Systems and Control Letters, vol. 8, pp. 161-171, 1986. 